\title{
EFFECT OF ANNEALING, STOICHIOMETRY, AND SURFACE ON MAGNETISM OF (Pr,Dy)FeCoB MICROPARTICLES ENSEMBLE
}

\begin{abstract}
Magnetic properties of powder ( $\mathrm{Pr}, \mathrm{Dy}) \mathrm{FeCoB}$ ferrimagnetic alloys and effects of annealing, surface states were analyzed. $\mathrm{X}$-ray photoelectron spectroscopy and Mössbauer spectra of powders indicate the effect of surface states on phase composition and magnetic properties of the studied powder, if particles average size is smaller than $10 \mu \mathrm{m}$. Effect of stoichiometry on magnetic anisotropy was found. Thermal stability of anisotropy field was proved by replacement of $\mathrm{Fe}$ atoms with Co atoms.

Keywords: Rare-earth metals, magnets, magnetisation, Mössbauer spectroscopy, X-ray spectroscopy
\end{abstract}

\section{Introduction}

The application of $\mathrm{FeCoB}$ based alloys attracts great attention of scientific community for purposes of the design of giant magnetoresistance (GMR) devices. Magnetic tunnelling junctions (MTJs) require perpendicular magnetic anisotropy to provide spin transfer switching in magnetic random access memory (MRAM) as well as in high density magnetic information devices. In fact, realization of low-dimensional and highreliability elements of computer memory requires thermal and magnetic stability of MTJs with perpendicular anisotropy [1,2].

Magnetic alloys based on Fe (Transition Metals, TM), rareearth transition metal (RE) and boron alloys such as NdFeB, [2] provide perpendicular magnetic anisotropy. The mechanism of perpendicular anisotropy in RE-TM-B alloys in crystalline and amorphous states has not been understood fully so far. Exchange interaction anisotropy, single-ion anisotropy, and dipole-dipole magnetic energies are convenient to explain physical origin of perpendicular anisotropy in the above mentioned materials [3-4]. Perpendicular anisotropy is sensitive to material and crystal structure, annealing, dipole-dipole interaction in the ensembles of microparticles, phase composition and surface electronic states. Our paper is focused on the analysis of these factors and their effect on magnetic properties of (Pr,Dy)FeCoB microstructured alloys.

In the recent publications it was shown that rare-earth atoms dope $\mathrm{FeCoB}$ alloys to enhance magnetic damping (Gilbert damping parameter). Possibly, such dopants have no quenched orbital moment and are selected in such a way that they increase damping. Examples of suitable rare-earth doping include $\mathrm{Ho}, \mathrm{Nd}$,
Sm, Dy, Pr, Tb. Er and Tm [5]. At present significantly increased magnetization in FeCoB alloys is hardly possible due to physical limitations. Angular momentum of ferrimagnetic rare-earth (RE) and transition metal (TM) lattices is compensated by the difference in RE and TM sublattice magnetic moments [6-9]. Interparticle interaction, surface magnetic anisotropy and surface layer of other chemical composition can strongly contribute to the Gilbert damping and angular moment of the alloys.

An additional reason for the development of the RE-TM-B magnets is their exceptional convenience for the creation of high thermally stable magnets of [10]. In RE-TM-B alloys, enhanced remanence and energy product result from exchange coupling between hard grains [11]. The exchange coupling affects coercivity. Substituents Pr and Dy change the exchange length. The Pr and Dy dopants are generally used to enhance exchange coupling and improve magnetic properties. The replacement of magnetic ions in TM sublattice with other ions allows one to discuss intra- and intersublattice exchange interactions and magnetic anisotropies.

Thus, our work was aimed at:

- $\quad$ Fabrication of (Pr,Dy)FeCoB microparticles of different composition and size,

- Study of contributions of particle size and morphology, dipole-dipole interaction, annealing and surface state effects to magnetic properties of the microparticles ensembles in the $2-370 \mathrm{~K}$ temperature range,

- Study of the effect of stoichiometry of Fe and Co atoms in the TM sublattice on the thermal stability of magnetic anisotropy field. 


\section{Experimental}

The alloys were melted in a high vacuum induction furnace. Argon flow spraying method was used to obtain dispersed melted drops of (Pr,Dy)FeCoB crystallized thereafter. Images of the particles obtained with a JSM-840 scanning electron microscope as well as with optical microscopy and size distribution are presented in Fig. 1a-c. Core-shell morphology of the microparticles is easy distinguishable in Fig. 1. Magnetic properties of the four samples (Table 1) differing in composition and particle size, were studied. Annealing of the samples was performed in vacuum furnace at $900^{\circ} \mathrm{C}$ during $30 \mathrm{~min}$.

Chemical composition was determined by two independent methods: atomic emission spectroscopy related to the bulk of the particles and X-ray photoelectron spectroscopy (XPS) related to the particles surface (Table 1). Bulk chemical composition of samples 1-4 was determined by a 730 ES atomic emission spectrometer. Surface $(\sim 1 \mathrm{~nm}$ depth) atomic concentrations of the elements were determined by XPS (Table 1). In the XPS experiments we used spectrometer equipped with a SPECS, PHOIBOS-Hsa 3500 energy analyzer. X-ray radiation was generated by $\mathrm{Mg}$ anode $(\mathrm{K} \alpha$ line at $1253.6 \mathrm{eV})$. Photoelectrons were collected by a $150 \mathrm{~mm}$ VSW hemispherical electron analyzer with $25 \mathrm{eV}$ pass energy. The full width at half maximum (FWHM) resolution was about $0.9 \mathrm{eV}$. All XPS spectra were acquired at normal emission. All binding energies of XPS spectra were referenced to the $\mathrm{C} 1 \mathrm{~s}$ peak at $284.6 \mathrm{eV}$.

TABLE 1

Chemical compositions and average sizes of particle in the samples 1-4

\begin{tabular}{|c|c|c|c|c|c|c|}
\hline \hline Sample & $\begin{array}{c}\text { Atomic } \\
\text { \% Pr }\end{array}$ & $\begin{array}{c}\text { Atomic } \\
\text { \% Dy }\end{array}$ & $\begin{array}{c}\text { Atomic } \\
\text { \% Fe }\end{array}$ & $\begin{array}{c}\text { Atomic } \\
\text { \% Co }\end{array}$ & $\begin{array}{c}\text { Atomic } \\
\text { \% B }\end{array}$ & $\begin{array}{c}\text { Particles } \\
\text { sizes, mm }\end{array}$ \\
\hline $\begin{array}{c}\text { №1 «bulk» } \\
\text { before annealing }\end{array}$ & 7.5 & 6.3 & 51.5 & 28 & 6.7 & $20-160$ \\
\hline $\begin{array}{c}\text { №2 «bulk» } \\
\text { before annealing }\end{array}$ & 7.5 & 6.3 & 51.5 & 28 & 6.7 & $0-10$ \\
\hline $\begin{array}{c}\text { №2 «surface» } \\
\text { before annealing }\end{array}$ & 21.35 & 12.19 & 40.94 & 19.01 & 6.5 & $0-10$ \\
\hline $\begin{array}{c}\text { №2 «surface» } \\
\text { after annealing }\end{array}$ & 18.88 & 9.62 & 45.66 & 21.27 & 4.56 & $0-10$ \\
\hline $\begin{array}{c}\text { №3 «bulk» } \\
\text { before annealing }\end{array}$ & 8.4 & 5.15 & 44.5 & 35 & 7 & $40-80$ \\
\hline $\begin{array}{c}\text { №4 «bulk» } \\
\text { before annealing }\end{array}$ & 8.4 & 5.15 & 44.5 & 35 & 7 & $0-10$ \\
\hline
\end{tabular}

b)

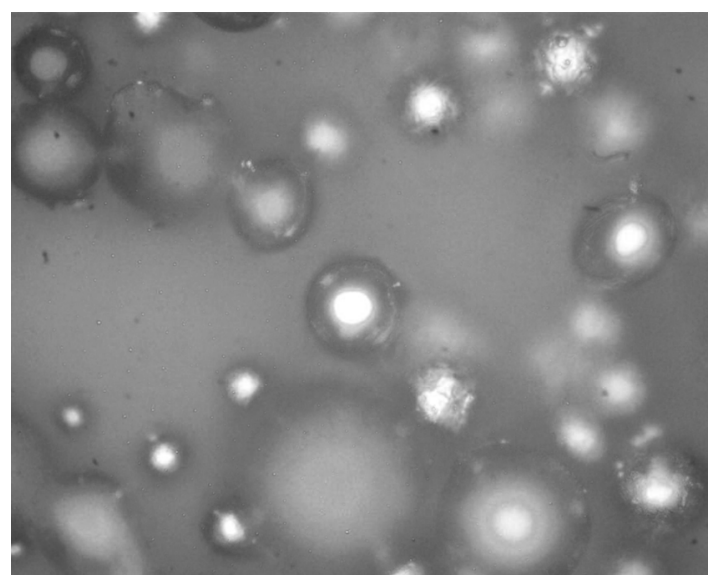

c)

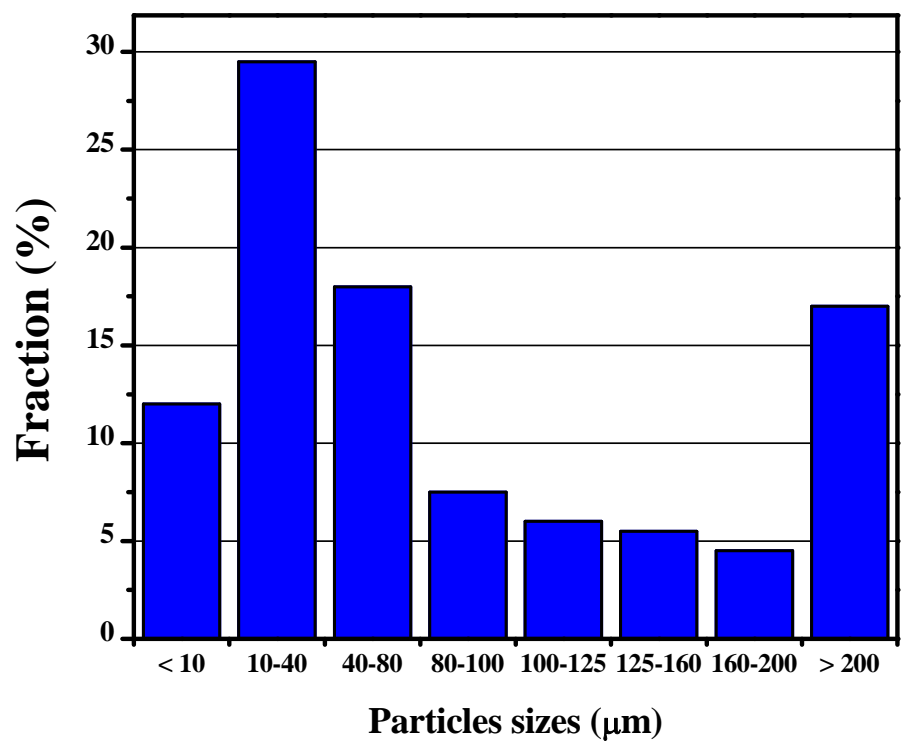

Fig. 1. SEM image of microparticles in "as grown" sample 2 (a), optical microscopy image (b), and size distribution of particles in sample 2 (c) 
X-ray diffraction spectra (XRD) were recorded by a DRON$3 \mathrm{M}$ spectrometer using Co $\mathrm{K}_{\alpha 12}$ irradiation. The $\theta-2 \theta$ spectra were recorded within $2 \theta=10^{\circ}-100^{\circ}$ range by Bragg-Brentano method at $300 \mathrm{~K}$. The recorded lines were indexed by the Match software using Crystallography Open Database. Spectrometer calibration was performed with a $\mathrm{SiO}_{2}$ sample.

Mössbauer spectra of ${ }^{57} \mathrm{Fe}$ in non-oriented samples were measured using ${ }^{57} \mathrm{Co} / \mathrm{Rh}$ source and a conventional constantacceleration spectrometer in zero external magnetic field at $290 \mathrm{~K}$. Spectrometer calibration was performed with $\alpha-\mathrm{Fe}$ sample. The hyperfine parameters were obtained by least-squares fitting to Lorentzian lines using the Mössbauer Software analysis.

Magnetization $M$ in constant magnetic field (DC) was measured by a superconducting quantum interference device (SQUID) magnetometer (MPMS 5XL Quantum design). The measurements were performed in the $2-370 \mathrm{~K}$ temperature range. Few different values of constant magnetic field from the range $H=0-50 \mathrm{kOe}$ were used. The $M(T)$ dependence was measured in the magnetic field $H$ between 5 Oe and 1000 Oe after cooling the sample from $370 \mathrm{~K}$ down to $2 \mathrm{~K}$ in zero field $(H<0.1 \mathrm{Oe})$ or in the $10 \mathrm{kOe}$ field, yielding the data in the zero-field cooled (ZFC) and the field-cooled (FC) conditions, respectively. Magnetization vs. magnetic field $M(H)$ was recorded both in the low-field conditions with $(H<100 \mathrm{Oe})$ for the correct estimation of the coercive force and in the normal-field conditions ( $H=0.1 \mathrm{kOe}-50 \mathrm{kOe})$. The real $\mathrm{m}$ ' and the imaginary $\mathrm{m}$ " parts of magnetization were studied in the alternating magnetic field (AC) of 2 Oe amplitude and $1-1400 \mathrm{~Hz}$ frequency at 2-370 $\mathrm{K}$.

\section{Results and discussion}

\subsection{X-ray and XPS analysis of "as grown" and annealed samples}

The XRD spectrum of annealed sample 2 shows contributions of the amorphous phase (wide halo), and 3 crystalline magnetic phases: $(\mathrm{Pr}, \mathrm{Dy})_{2}(\mathrm{Fe}, \mathrm{Co})_{14} \mathrm{~B}(36 \%),(\mathrm{Pr}, \mathrm{Dy})(\mathrm{Fe}, \mathrm{Co})_{4} \mathrm{~B}(32 \%)$ and $\mathrm{DyFe}_{5}(31 \%)$. Corresponding lines and reflection planes are marked in Fig. 2a. Similar spectra were observed for the (Pr,Dy) FeCoBZr alloys [12]. Few weak additional lines at $2 \Theta=26^{\circ}$ correspond to $\alpha-\mathrm{Fe}$ [13]. Their contribution does not exceed $1 \%$. In the "as grown" sample, one can observe two series of lines strongly differing in width (Fig. 2a). Linewidth of a XRD spectrum depends on particle size. Broadened XRD lines are usually observed when crystallite size is smaller than $0.5 \mu \mathrm{m}$. If bimodal size distribution is taken into account (Fig. 1), the widening can be explained by the presence of small and large particles.

Annealing strongly affects the XRD spectrum (Fig. 2b). Halo corresponding to the amorphous phase disappears, while the other lines are narrowed and their intensities are increased. The decomposition of the XRD spectrum is presented in Fig. $2 b$. Phase composition of sample $\mathbf{2}$ changes after annealing: amount of $(\mathrm{RE})_{2}(\mathrm{TM})_{14} \mathrm{~B}$ phase increases up to $92 \%$, while phase $\mathrm{DyFe}_{5}$ disappears. The other lines correspond to $\mathrm{RETM}_{4} \mathrm{~B}$ phase (7\%) and $\alpha-\mathrm{Fe}$ (up to $1 \%$ ) (Fig. 2b). Their contributions to the spectrum in the annealed samples were small unlike those for the "as grown" samples.
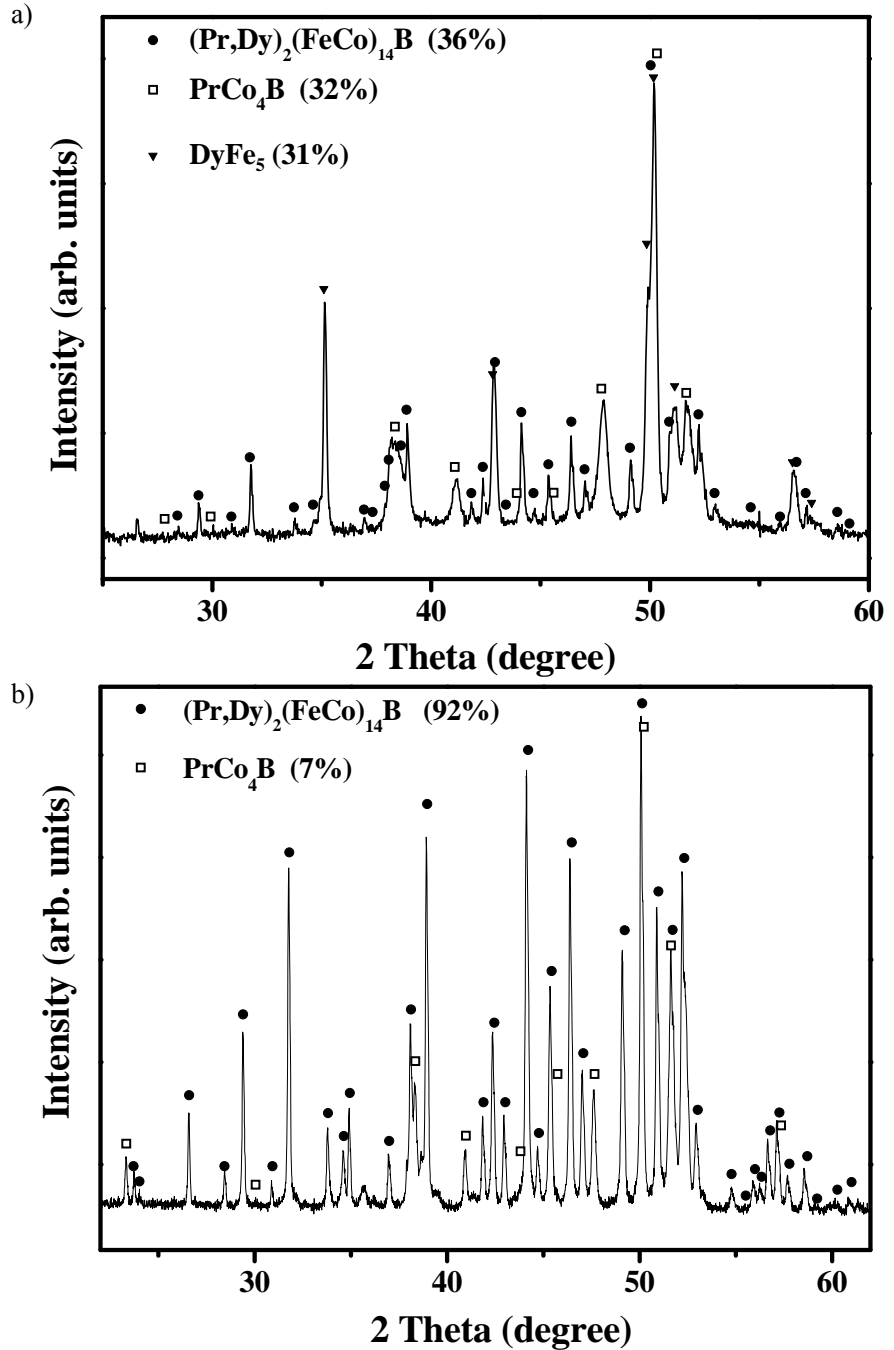

Fig. 2. Survey X-ray diffraction spectra for sample 2 before (a) and after (b) annealing at $900^{\circ} \mathrm{C}$

The survey XPS spectra for "as grown" and annealed sample 2 are presented in Fig. 3. They contain core-shell lines of the $\operatorname{Pr}(3 d), \operatorname{Co}(2 p), F e(2 p), \operatorname{Dy}(4 d)$, and B(1s) levels. Atomic concentrations of the atoms estimated from the XPS spectra differ from the bulk values determined by atomic emission technique (Table 1). Surface concentrations changed under annealing. The decrease of surface concentration of mobile boron was observed after annealing as well as slight decrease of Dy and Pr concentrations. Concentrations of $\mathrm{Fe}$ and $\mathrm{Co}$ atoms were found to be higher after annealing. Obvious splitting of the core-shell Fe line (the step at $E=707 \mathrm{eV}$ binding energy) corresponds to $\mathrm{Fe}$ oxide phases on the surface $[14,15]$. Two lines at $726 \mathrm{eV}$ and 712 $\mathrm{eV}$ in the $\mathrm{Fe}$ spectrum (see insert in Fig. 3) correspond to $\mathrm{Fe}^{3+}$ and $\mathrm{Fe}^{2+}$ ions, respectively. Thus, surface chemical composition is different as compared with bulk composition. Therefore, in small 
a)

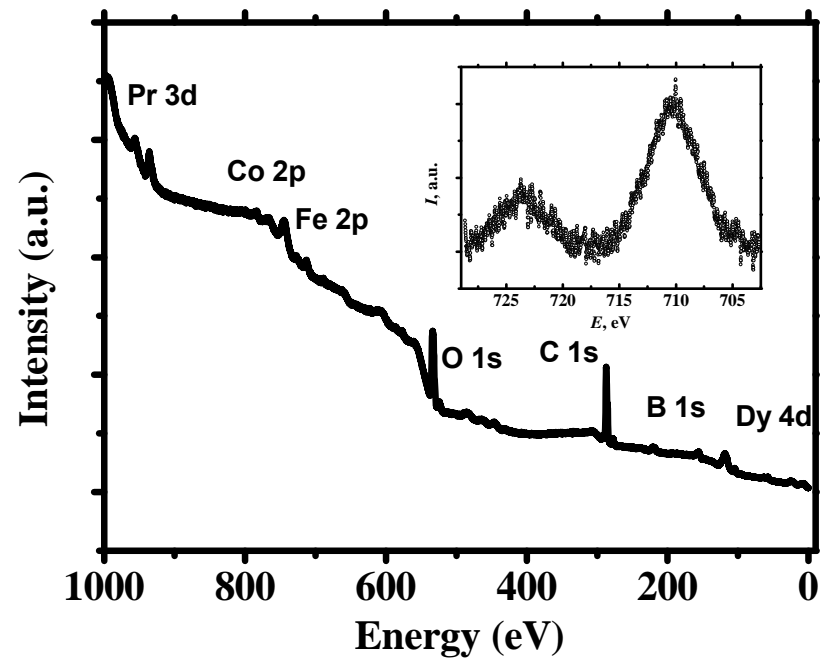

b)

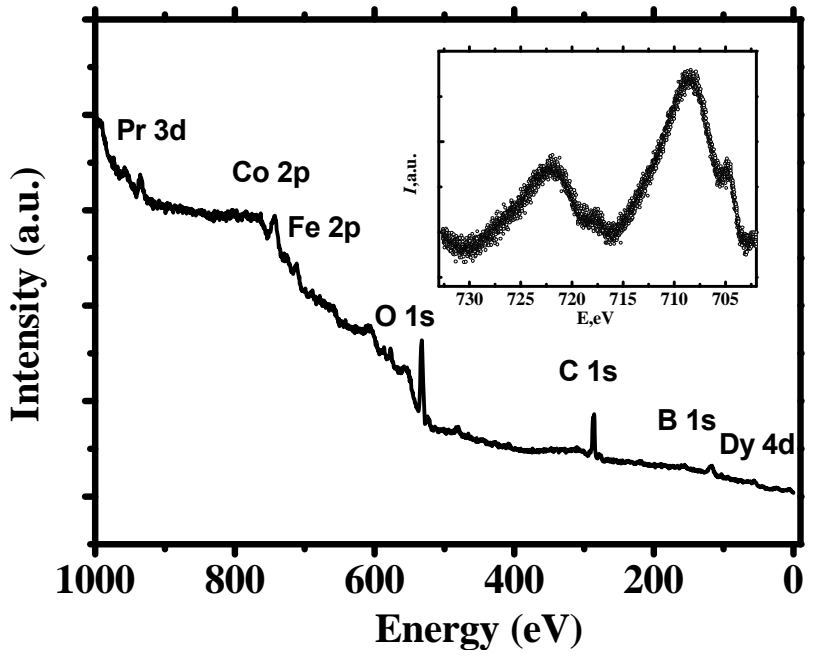

Fig. 3. Survey XPS spectrum of the surface of sample 2 at $300 \mathrm{~K}$ : (a) before and (b) after annealing. Core level spectra of the $\mathrm{Fe} 2 \mathrm{p}$ level are shown in inserts

particles of 0.1-10 $\mu \mathrm{m}$ size, one can expect a more significant contribution of the surface states to their magnetic properties than one expected in the large particles.

\subsection{Effects of annealing and particle size on magnetic properties}

Shapes of magnetic hysteresis loops were similar in a series of samples. The hysteresis loop for as grown sample 2 at $T=2 \mathrm{~K}$ (coercive force $H_{\mathrm{c}}=0.71 \mathrm{kOe}$, saturation magnetization $\left.M_{\text {sat }}=1.1 \mu_{\mathrm{B}}\right)$ is presented in Fig. 4a. Calculations of $M_{\text {sat }}$ per formula unit were performed taking into account typical effective magnetic moments of the $\operatorname{Pr}\left(3.3 \mu_{\mathrm{B}}\right)$, Dy $\left(10 \mu_{\mathrm{B}}\right)$, Fe $\left(2.2 \mu_{\mathrm{B}}\right)$, and $\mathrm{Co}\left(1.5 \mu_{\mathrm{B}}\right)$ ions [1]. If one assumes opposite directions of the local effective magnetic moment of Dy ions relative to the effective moments of the other atoms $(\mathrm{Pr}, \mathrm{Fe}, \mathrm{Co})$, the calculation of the expected theoretical value gives $\mu_{\text {eff }}=1.106$ $\mathrm{m}_{\mathrm{B}}$ close to experimentally determined $\mu_{\text {eff }}$ (shown by dashed horizontal line in Fig. 4a). In our calculations we used relative concentrations of chemical elements in the bulk determined by atomic emission spectroscopy (Table 1). If we take into account surface concentrations determined by XPS, the calculation of the expected effective magnetic moment gives $\mu_{\text {eff }}=0.62 \mu_{\mathrm{B}}$ that is lower than the experimental value (shown by solid horizontal line in Fig. 4a). The strong deviation of surface magnetic state and chemical composition from those measured in the bulk of the particles is observed. A similar difference between bulk and surface chemical compositions and corresponding effective magnetic moments was observed for samples $\mathbf{1}, \mathbf{3}$ and $\mathbf{4}$.

At $T<100 \mathrm{~K}$ the hysteresis loop was of "butterfly" type, while normal shape of the loops was observed at high temperatures. Magnetic hysteresis loop for "as grown" sample 2 at $T=300 \mathrm{~K}$ is shown as curve A in Fig. $4 \mathrm{~b}$. At $300 \mathrm{~K}$ sample 2 has approximately same saturation magnetization $M_{\mathrm{S}}=1.1 \mu_{\mathrm{B}}$ as at $T=2 \mathrm{~K}$, but coercive force drops from $0.71 \mathrm{kOe}$ at $2 \mathrm{~K}$ to $H_{\mathrm{c}}=0.41 \mathrm{kOe}$ at $300 \mathrm{~K}$.

a)

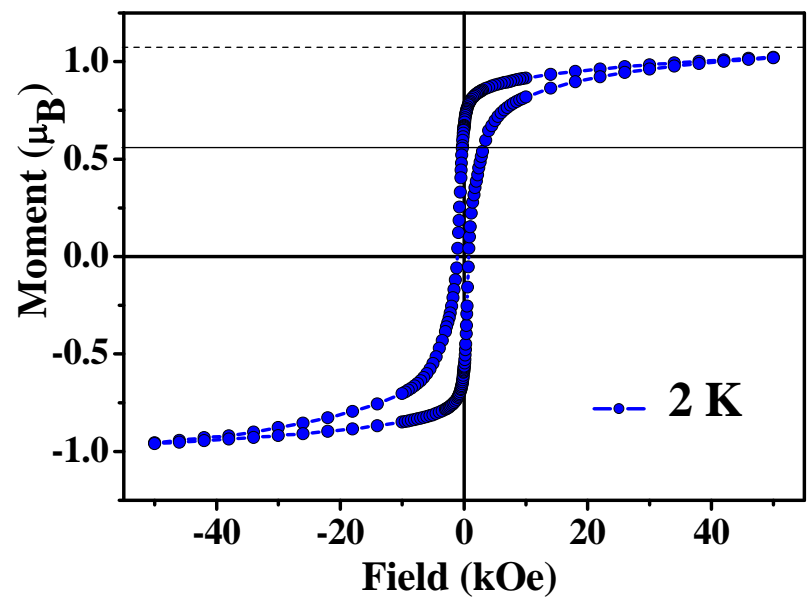

b)

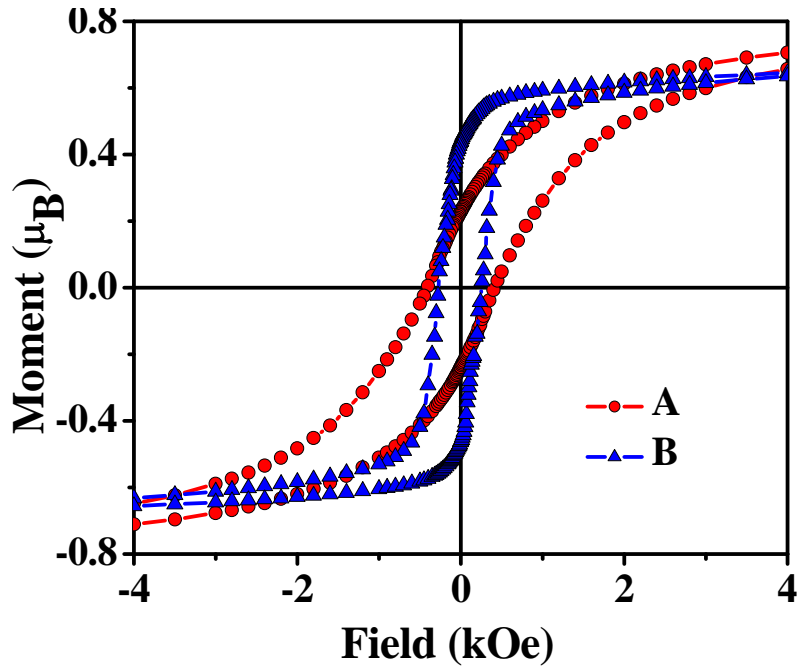

Fig. 4. Magnetic hysteresis of sample 2: (a) at $T=2 \mathrm{~K}$ before annealing, (b) at $300 \mathrm{~K}$ before annealing (circles) and after annealing (triangles). Calculated $\mu_{\text {eff }}$ value for bulk and surface atomic concentrations are shown by dashed and solid horizontal lines, respectively.

We also studied the effect of annealing on magnetic properties of samples 1-4. Each sample was annealed at $T=900^{\circ} \mathrm{C}$ for 30 minutes. Magnetic hysteresis loop of the annealed sample 2 
is presented as curve $\mathbf{B}$ in Fig. 4b. The annealing of sample 2 results in the decrease of coercive force to $0.25 \mathrm{kOe}$. Saturation magnetization $M_{\text {sat }}$ was not sensitive to annealing.

The temperature dependencies of magnetization parameters are shown in Fig. 5. The temperature dependencies of coercive force were identical in the series of the samples: $H_{\mathrm{c}}$ decreased as the samples were heated up to $370 \mathrm{~K}$. Above this temperature

a)

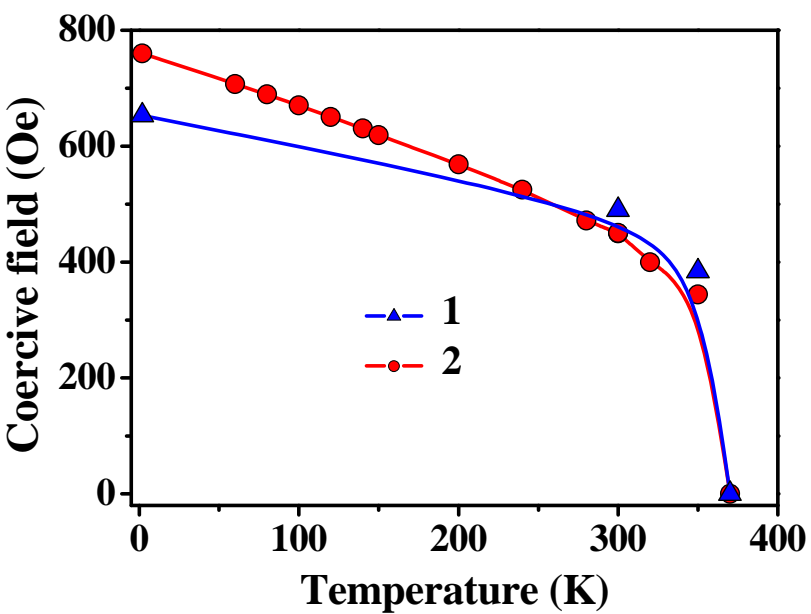

b)

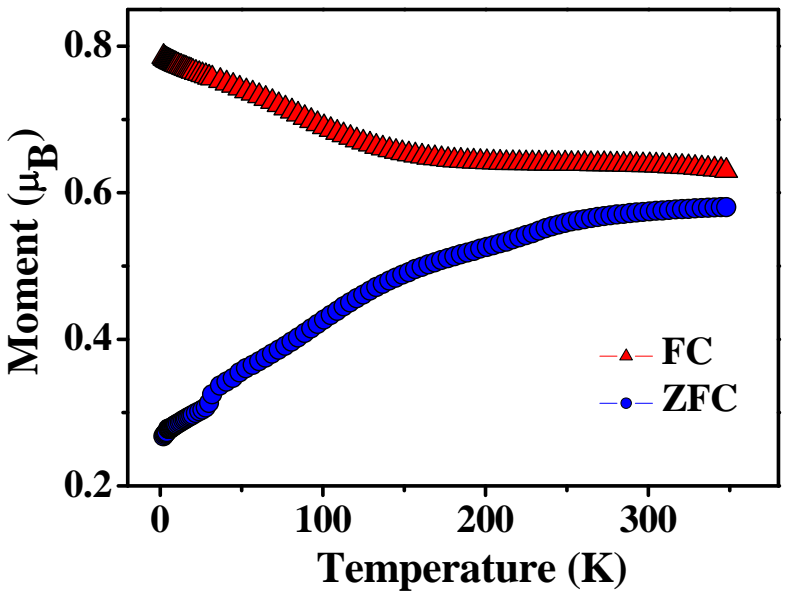

c)

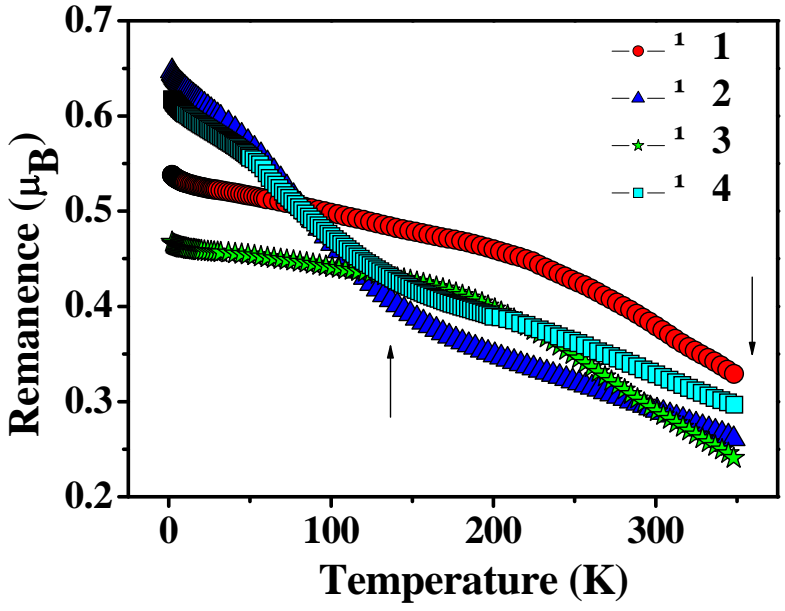

Fig. 5. Temperature dependencies of: (a) coercive force $H_{\mathrm{c}}$ of samples 1 and 2, (b) magnetization $M$ of sample 2 cooled in $10 \mathrm{kOe}$ magnetic field (FC, triangles) and zero magnetic field (ZFC, circles). Measurements were performed in $1 \mathrm{kOe}$ magnetic field, (c) remnant magnetization $M_{\text {rem }}$ of samples 1-4 after cooling down to $2 \mathrm{~K}$ in $50 \mathrm{kOe}$ magnetic field (numeration of the curves corresponds to that of the samples)
$H_{\mathrm{c}}$ becomes zero (Fig. 5a). Large size of the particles provides high blocking temperature estimated from the divergence of Field Cooling (FC) and Zero Field Cooling (ZFC) curves measured in magnetic field of $1 \mathrm{kOe}$ after the sample was cooled down to $2 \mathrm{~K}$ in $5 \mathrm{~T}$ magnetic field (Fig. 5b). Although the SQUID magnetometer did not cover temperatures higher than $370 \mathrm{~K}$, one could estimate blocking temperature by extrapolation of the FC and ZFC curves to 390-400 K.

The temperature dependencies of remnant magnetic moments $M_{\text {rem }}$ for samples 1-4 were obtained from measurements in zero magnetic field using the samples previously cooled down to $2 \mathrm{~K}$ in magnetic field of $5 \mathrm{~T}$ (Fig. 5c). The measured dependencies $M_{\text {rem }}(T)$ can be grouped in two identical pairs: dependencies for samples 1 and 3 (large particles $<160 \mu \mathrm{m}$ ) and dependencies $M_{\text {rem }}(T)$ for samples $\mathbf{2}$ and $\mathbf{4}$ (small particles $<10 \mu \mathrm{m}$ ) were identical (Fig. 5c). These data are in a good agreement with the XPS results indicating modified composition of the surface layers (Table 1) responsible for the additional contribution to magnetization. One can observe Bloch character of $M_{\text {rem }}(T)=M_{0}\left(1-T / T_{\mathrm{c}}\right)^{3 / 2}$ in samples 1 and 3 (large particles), while a superposition of the twice repeated Bloch dependencies corresponding to two critical temperatures $T_{\mathrm{c} 1}=150 \mathrm{~K}$ and $T_{\mathrm{c} 2}=390 \mathrm{~K}$ can be distinguished in the small particles of samples 2 and 4 (Fig. 4c). The low-temperature amorphous phase mentioned in [16] has $T_{\mathrm{c}}=170 \mathrm{~K}$ close to $T_{\mathrm{c} 1}=150 \mathrm{~K}$ determined in our experiments. This phase which is probably present in the surface layers could explain the double Curie point. The other possible explanation is spin-flop transition due to the competition of surface and crystalline magnetic anisotropy of the particles. Tilting of the main axis may result in changes in the longitudinal magnetization projection measured by a SQUID magnetometer. Measurements on polycrystalline materials performed by various authors indicated that several $\mathrm{RE}_{2} \mathrm{Fe}_{14} \mathrm{~B}$ compounds give rise to changes in magnetic structure when cooled from the Curie temperature down to $4.2 \mathrm{~K}$ [17-20]. More detailed information regarding these structural changes was obtained from measurements on single crystals. Givord et al. [21] showed that at $4.2 \mathrm{~K}$ the spontaneous magnetization direction is intermediate between the $c$ axis and the basal plane.

\subsection{Mössbauer spectroscopy}

Mössbauer spectra of all the samples were recorded at 300 K. Fig. 6 shows the spectra of "as grown" sample 1 (Fig. 6a), "as grown" sample 2 (Fig. 6b) and annealed sample 2 (Fig. 6c). Allowed transitions between the doublet of the ground nuclear state of ${ }^{57} \mathrm{Fe}(\operatorname{spin} I=1 / 2)$ and the triplet of the excited nuclear state ( $\operatorname{spin} I=3 / 2$ ) result in a sextet since the sample is ferromagnetic at room temperature. For that reason, Fermi interaction (average magnetic field) splits the doublet and the triplet providing six allowed transitions $\Delta I= \pm 1$ between nuclear energy levels. All the recorded spectra were decomposed by six sextets and one doublet. The doublet corresponds to a contribution of a small amount of paramagnetic impurity. Six sextets were used due 
a)

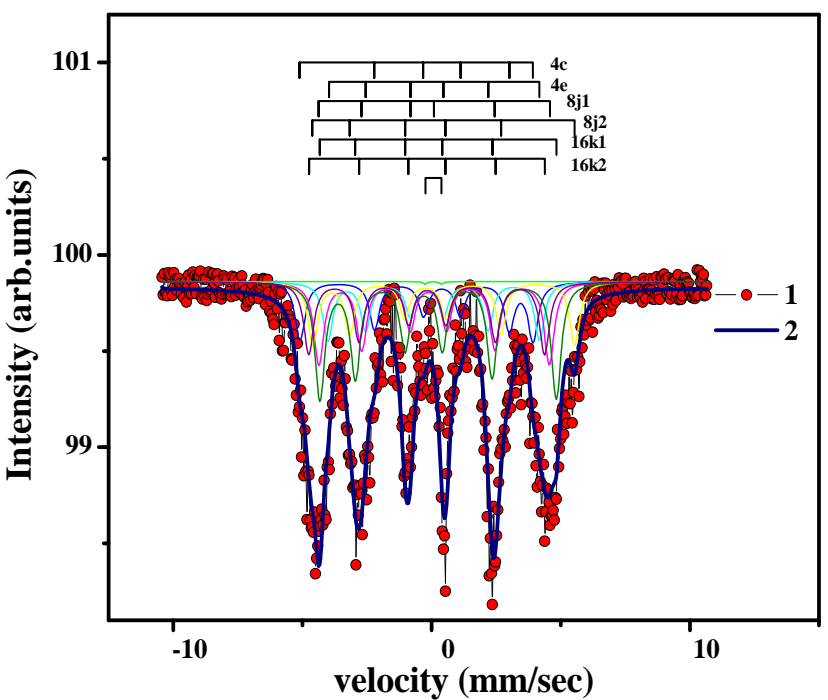

b)

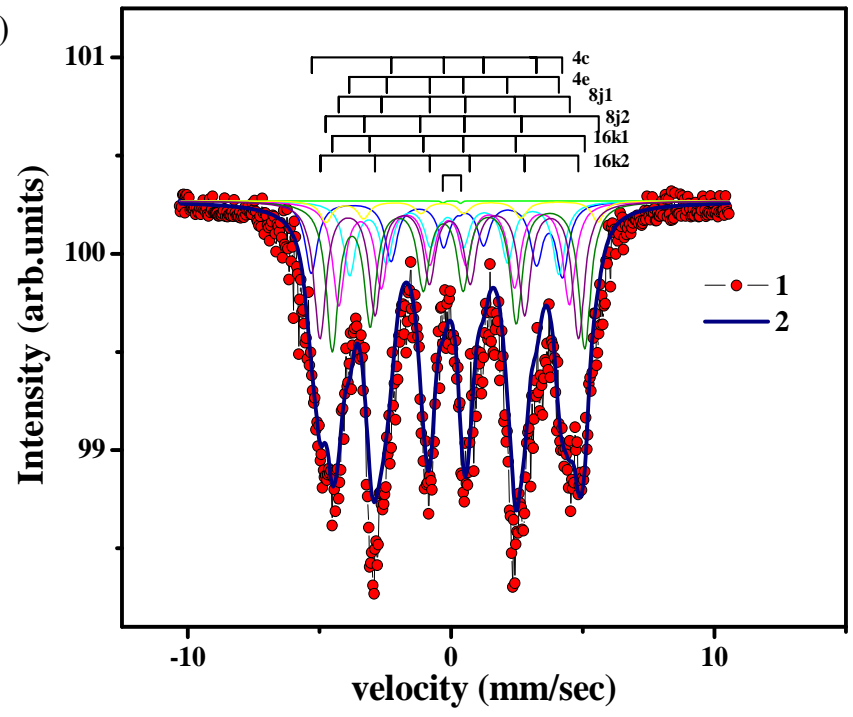

c)

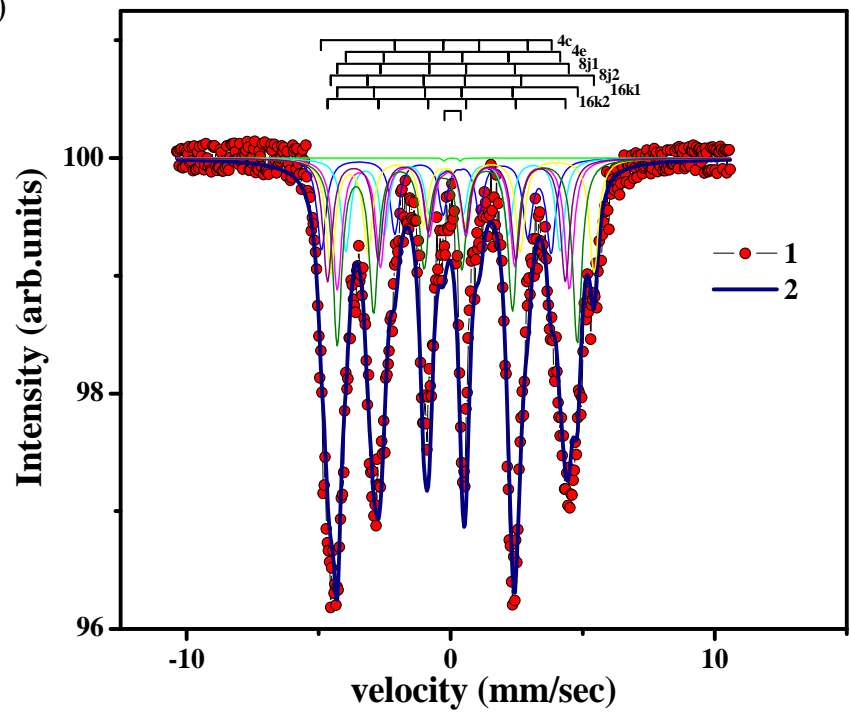

Fig. 6. Mossbauer spectra recorded at $T=300 \mathrm{~K}$ in the absence of magnetic field: (a) in "as grown" sample 1, (b) in "as grown" sample 2 , (c) in annealed sample 2 . On all spectra circular symbols correspond to experimental data, thick solid line is approximation of spectrum by the sum of doublet $\left((\mathrm{Pr}, \mathrm{Dy})(\mathrm{Fe}, \mathrm{Co})_{4} \mathrm{~B}\right.$ paramagnetic phase $)$ and six sextets (thin lines), corresponding to six non-equivalent $\mathrm{Fe}$ positions in the ferromagnetic 2-14-1 phase to that six non equivalent $\mathrm{Fe}$ positions are presented in the crystalline structure of the $(\mathrm{Pr}, \mathrm{Dy})_{2}(\mathrm{FeCo})_{14} \mathrm{~B}$ phase [1].

A comparison of the spectra for samples 1 and $\mathbf{2}$ with that of reference a- Fe shows that there is a longer distance between outer lines of the sextet. The distance is proportional to the quadrupole splitting $\mathrm{Q}$ and magnetization of the sample. The effect of longer distance between outer lines as compared with inner lines of the sextet indicates that symmetry of Fe nuclei environment differs from cubic one as it was expected for $\mathrm{FeCoB}$ based alloys in which cubic symmetry is distorted by boron.

The approximation of the spectrum of the "as grown" sample showed that concentration of a- Fe did not exceed 2-3\% that is in agreement with XRD analysis. In the annealed samples concentration of a- Fe increased by more than 5\% (a small step appeared on the external wing of the sextet).

A comparison of the spectra of samples 1 and $\mathbf{2}$ indicates changes in local magnetic fields on the ${ }^{57} \mathrm{Fe}$ nuclei. The changes are most probably due to the contribution of the surface layers to the Mössbauer spectra as it was detected by the XPS method (see paragraph 3.1). Remarkable broadening of the lines in comparison with the reference sample allows us to conclude on the presence of few phases that is in a good agreement with the results of XRD analysis (see paragraph 3.1). The comparison of Fig. $6 \mathrm{~b}$ and Fig. $6 \mathrm{c}$ allows a conclusion on lines narrowing after annealing and the appearance of the perfect phase. This fact is also supported by XRD analysis.

In the next series of the experiments the dependence of the population of atomic sites in the TM sublattice versus Co concentration $x$ was studied. A series of the powder samples with varied Co concentration was measured by Mössbauer technique. The experimental spectra were decomposed as it was described above. Integral contributions of each sextet results in relative concentration of $\mathrm{Fe}$ ions in the corresponding atomic sites of the TM sublattice. The examples of populations of atomic sites are shown in Fig. 7 for different concentrations of Co. One can observe that the growth of average Co concentration initiates progressive $\mathrm{Fe}$ depopulation of atomic sites $16 \mathrm{k}_{1}, 16 \mathrm{k}_{2}, 8 \mathrm{j}_{1}$. At the same time the increase of Fe populations of $8 \mathrm{j}_{2}, 4 \mathrm{e}$ and $4 \mathrm{c}$ sites was observed. The last ones have RE atoms on the nearest neighbor positions. The redistribution of the Fe atoms in the TM sublattice remarkably improves temperature stability of anisotropy field $H_{\mathrm{A}}$ (Fig. 7).

Best approximation of the $H_{\mathrm{A}}(T)$ dependence was found by the empirical equation described in [22]:

$$
H_{\mathrm{A}}(T)=\mathrm{A} \exp \left(-\alpha T^{2}\right)+\mathrm{C}
$$

The results of the approximation for $x=0.52$ and $x=0.83$ samples are shown by solid lines in Fig. 8. The parameters similar to those described in [23] were determined in $x=0.52$ sample $\left(\mathrm{C}=132 \times 10^{4} \mathrm{erg} / \mathrm{cm}^{3} \pm 8 \times 10^{4} \mathrm{erg} / \mathrm{cm}^{3}, \alpha=710^{-5} \mathrm{~K}^{-2}\right.$, $\left.\mathrm{A}=82 \times 10^{4} \mathrm{erg} / \mathrm{cm}^{3} \pm 8 \times 10^{4} \mathrm{erg} / \mathrm{cm}^{3}\right)$ as well as in $x=0.83$ sample $\left(\mathrm{C}=130 \times 10^{4} \mathrm{erg} / \mathrm{cm}^{3} \pm 6 \times 10^{4} \mathrm{erg} / \mathrm{cm}^{3}, \alpha=410^{-5} \mathrm{~K}^{-2}\right.$, $\left.\mathrm{A}=32 \times 10^{4} \mathrm{erg} / \mathrm{cm}^{3} \pm 6 \times 10^{4} \mathrm{erg} / \mathrm{cm}^{3}\right)$. Here $\mathrm{C}$ is a part of anisotropy independent on Co concentration $x$. This part probably corresponds to RE sublattice. Parameters $\alpha$ and A decreased 
a)

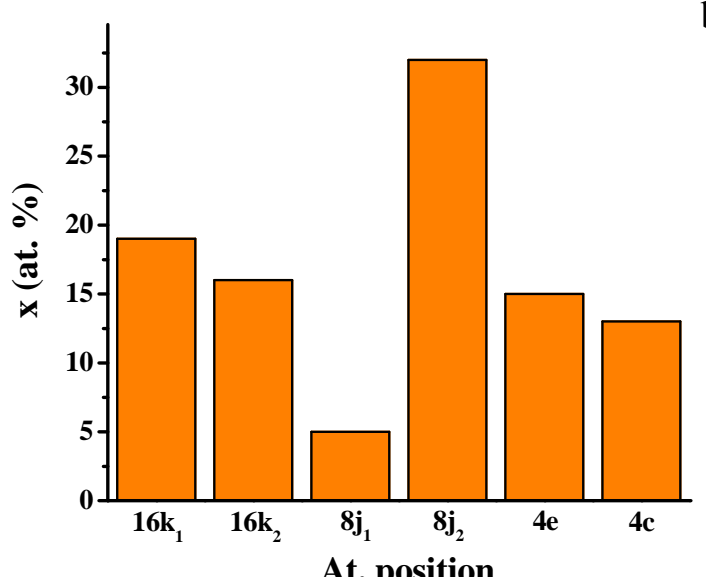

b)

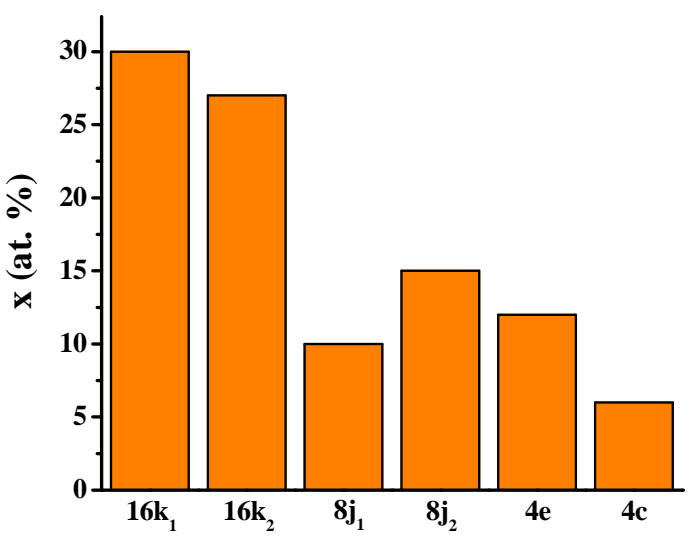

At. position

c)

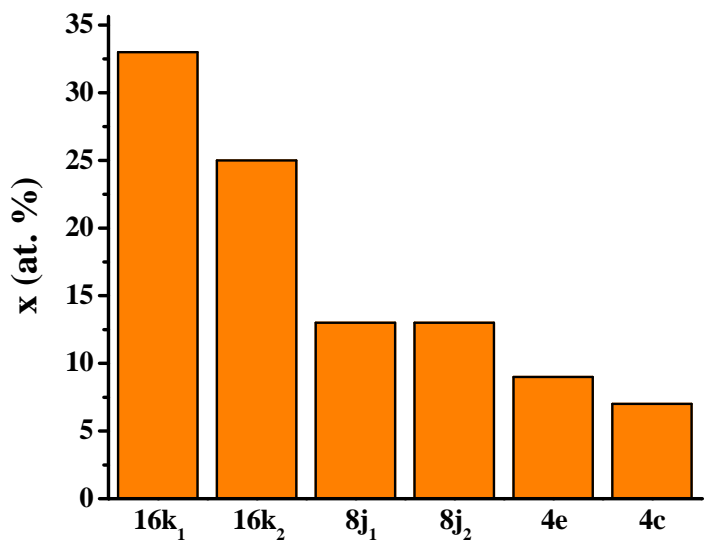

At. position

Fig. 7. Populations of the atomic sites in the $\mathrm{TM}$ sublattice by $\mathrm{Fe}$ atoms in series of the samples with progressively increased $\mathrm{Fe}$ content: (a) $\mathrm{Pr}_{2}\left(\mathrm{Fe}_{0.17} \mathrm{Co}_{0.83}\right)_{14} \mathrm{~B}$, (b) $\left(\mathrm{Pr}_{0.68} \mathrm{Dy}_{0.32}\right)_{2}\left(\mathrm{Fe}_{0.53} \mathrm{Co}_{0.47}\right)_{14} \mathrm{~B}$, (c) $\left(\mathrm{Pr}_{0.39} \mathrm{Dy}_{0.41} \mathrm{Gd}_{0.20}\right)_{2}\left(\mathrm{Fe}_{0.69} \mathrm{Co}_{0.31}\right)_{14} \mathrm{~B}$

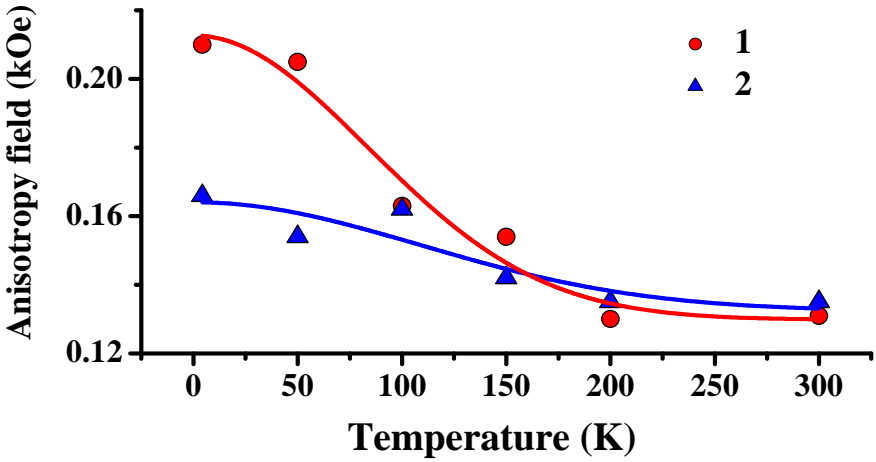

Fig. 8. Temperature dependence of anisotropy field $H_{\mathrm{A}}$ in the samples (1) $\left(\mathrm{Pr}_{0.70} \mathrm{Dy}_{0.30}\right)_{15,3}\left(\mathrm{Fe}_{0.48} \mathrm{Co}_{0.52}\right)_{70.7} \mathrm{~B}_{14.0}$, (2) $\left(\mathrm{Pr}_{0.85} \mathrm{Dy}_{0.15}\right)_{16.5}\left(\mathrm{Fe}_{0.17}\right.$ $\left.\mathrm{Co}_{0.83}\right)_{69.4} \mathrm{~B}_{14.1}$. Solid lines are approximations described in the text

almost twice as Co concentration was changed from $x=0.52$ up to $x=0.83$.

Another possible explanation is based on the fact that increasing Co concentration leads to the increase of boron enriched phases (for example, $\mathrm{RETM}_{4} \mathrm{~B}$ ) [24]. These phases stimulate both intra- and interphase boron diffusion [25]. Since in our experiments Co concentration was much higher than previously published by other authors [26-28], one can expect a strong enhancement of B diffusion and oversaturation of the main $\mathrm{RE}_{2} \mathrm{TM}_{14} \mathrm{~B}_{1}$ phase by boron. The replacement of $\mathrm{Fe}$ atoms by $\mathrm{B}$ is only one way for oversaturation of the phases. Thus, the replacement of boron nonmagnetic atoms by Fe atoms decreases exchange interaction between RE and TM sublattices. Since RE sublattice is a main source of magnetic anisotropy and its temperature dependence, the unlinking of the RE and TM sublattices leads to temperature independent behavior of $H_{\mathrm{A}}$ (Fig. 8). A similar effect was observed in the Laves phase [29].

\section{Conclusions}

- The presence of the $(\mathrm{Pr}, \mathrm{Dy})_{2}(\mathrm{FeCo})_{14} \mathrm{~B},(\mathrm{Pr}, \mathrm{Dy})(\mathrm{Fe}, \mathrm{Co})_{4} \mathrm{~B}$, $\alpha-\mathrm{Fe}, \mathrm{DyFe}_{5}$, phases in the microparticles of $(\mathrm{Pr}, \mathrm{Dy}) \mathrm{FeCoB}$ prepared by gas spraying method was identified. Vacuum annealing at $900^{\circ} \mathrm{C}$ provides crystallization of the amorphous $(\mathrm{Pr}, \mathrm{Dy})_{2}(\mathrm{FeCo})_{14} \mathrm{~B}$ phase dominating in the annealed samples (up to $92 \%$ ).

- In the powders of $10 \mu \mathrm{m}$ upper limit of particle size, the low-temperature phase $\left(T_{\mathrm{c}}=150 \mathrm{~K}\right)$ contributes to their magnetic properties in contrast with a series of samples of $100 \mu \mathrm{m}$ upper limit of particle size.

- The difference between surface (1 $\mathrm{nm}$ depth) and bulk chemical composition of the samples and changes in surface 
composition after annealing were detected by XPS technique. The volume fraction of the surface layers of modified chemical composition is compatible with the bulk fraction as it was determined from electron microscopy. Surface layers contribute strongly to the magnetic properties of small particles $(<10 \mu \mathrm{m}$ in size $)$ and their Mossbauer spectra. Large particles (up to $80 \mu \mathrm{m}$ in size) show no contribution of the surface layers to their magnetic properties.

- The changes in stoichiometry of Fe and Co in TM sublattice lead to the insertion of Co atoms to the Fe sites. These replacements unlink exchange interaction between RE and TM sublattices suppressing temperature dependence of magnetic anisotropy.

\section{Acknowledgements}

This work was financially supported by Ministry of Education and Science of Russian Federation grant 3.1992.2017/ПЧ.

\section{REFERENCES}

[1] A.V. Khvalkovskiy, D. Apalkov, S. Watts, R. Chepulskii, R.S. Beach, A. Ong, X. Tang, A. Driskill-Smith, W.H. Butler, P.B. Visscher, D. Lottis, E. Chen, V. Nikitin, M. Krounbi. Basic principles of STT-MRAM cell operation in memory arrays. J. Phys. D: Appl. Phys. 46, 074001 (2013).

[2] J.F. Herbst. R2Fe14B materials: Intrinsic properties and technological aspects. Rev. Mod. Phys. 63, 819 (1991).

[3] Y. Suzuki, J. Haimovich, T. Egami. Bond-orientational anisotropy in metallic glasses observed by x-ray diffraction. J. Phys. Rev. B 35, 2162 (1987).

[4] S. Bae, N. Thiyagarajah. Developments in Giant Magnetoresistance and Tunneling Magnetoresistance Based Spintronic Devices with Perpendicular Anisotropy. Magnetic Thin Films: Properties, Performance and applications 4, 135 (2011).

[5] M.W. Covington et al., Damping control in magnetic recording systems (Patent US 2007/0003792) (2007).

[6] R.K. Wangsness. Sublattice Effects in Magnetic Resonance. J. Phys. Rev. 91, 1085 (1953).

[7] R. Giles, M. Mansuripur.Dynamics of magnetization reversal in amorphous films of RE-TM alloys. J. Magn. Soc. Jpn. 15-S1, 299 (1991).

[8] T. Kato, K. Nakazawa, R. Komiya, N. Nishizawa, S. Tsunashima, S. Iwata. Compositional Dependence of g-Factor and Damping Constant of GdFeCo Amorphous Alloy Films. IEEE Trans. on magnetics 44, 3380 (2008).

[9] R.F. Soohoo, A.H. Morrish. FMR measurement of anisotropy dispersion in amorphous GdFe films. J. Appl. Phys. 50, 1639 (1979).

[10] K.J. Strnat. Modern permanent magnets for applications in electro-technology. Proceedings of the IEEE 78, 923 (1990).

[11] Z.W. Liu, Y. Liu, P.K. Deheri, R.V. Ramanujan, H.A. Davies. Improving permanent magnetic properties of rapidly solidified nanophase RE-TM-B alloys by compositional modification. J. Magn. Magn. Mat. 321, 2290 (2009).
[12] P. Pawlik, H.A. Davies. Glass formability of Fe-Co-Pr-Dy-Zr-B alloys and magnetic properties following devitrification. Scripta Materialia 49, 755 (2003).

[13] Z.Q. Jin, N.N. Thadhani, M. McGill, Y. Ding, Z.L. Wang, M. Chen, H. Zeng, V.M. Chakka, J.P. Liu. Explosive shock processing of $\mathrm{Pr}_{2} \mathrm{Fe}_{14} \mathrm{~B} / \alpha-\mathrm{Fe}$ exchange-coupled nanocomposite bulk magnets. J. Mat. Res. 20, 599 (2005).

[14] M.C. Biesinger, B.P. Payne, A.P. Grosvenor, L.W.M. Lau, A.R. Gerson, R.St.C. Smart. Resolving surface chemical states in XPS analysis of first row transition metals, oxides and hydroxides: $\mathrm{Cr}$, Mn, Fe, Co and Ni. Appl. Surf. Scien. 257, 2717 (2011).

[15] I. Uhlig, R. Szargan, H.W. Nesbitt, K. Laajalehto. Surface states and reactivity of pyrite and marcasite. Appl. Surf. Scien. 179, 222 (2001)

[16] J. Yin, B. Shen, D. Wang, X. Yin, M. Wang, Y. Du. Magnetic properties and magnetic domain structure of $\mathrm{Nd}_{6} \mathrm{Dy}_{2} \mathrm{Fe}_{82} \mathrm{Co}_{4} \mathrm{~B}_{6}$ nanocomposite magnets. J. Alloys Compd. 316, 296 (2001).

[17] S. Sinnema, R.J. Radwanski, J.J.M. Franse, D.B. de Mooij, K.H.J. Buschow. Magnetic properties of ternary rare-earth compounds of the type $\mathrm{R}_{2} \mathrm{Fe}_{14}$ B. J. Magn. Magn. Mat. 44, 333 (1984).

[18] F. Spada, C. Abache, H. Oesterreicher. Crystallographic and magnetic properties of rare earth-transition metal compounds based on boron. J. Less-Common Met. 99, L21 (1984).

[19] R. Grössinger, P. Obitsch, X.K. Sun, R. Eibler, H.R. Kirchmayr, F. Rothwarf, H. Sassik. The anisotropy of $\mathrm{Nd}_{2} \mathrm{Fe}_{14} \mathrm{~B}$ magnets. Mat. Lett. 2, 539 (1984).

[20] H. Oesterreicher. On the Spin Reorientation in $\mathrm{R}_{2} \mathrm{Fe}_{14} \mathrm{~B}$ Compounds. Phys. Stat. Sol. (B) 131, K123 (1985).

[21] D. Givord, H.S. Li, R.P. de la Bâthie. Magnetic properties of $\mathrm{Y}_{2} \mathrm{Fe}_{14} \mathrm{~B}$ and $\mathrm{Nd}_{2} \mathrm{Fe}_{14} \mathrm{~B}$ single crystals. Sol. Stat. Com. 51, 857 (1984).

[22] N.L. Bryukhatov, L.V. Kirenskil. Temperature effect on magnetic anisotropy energy of ferromagnetic crystals. J. Exp. Theor. Phys. (U.S.S.R.) 8, 198 (1938).

[23] A.I. Drokin, L.I. Slobodskoi. Concerning the Temperature Dependence of the Magnetic Anisotropy Constants of Ferromagnets and Ferrites. J. Exp. Theor. Phys. (U.S.S.R.) 51, 1023 (1967).

[24] C.N. Christodoulou, T.B. Massalski, W.E. Wallance. Liquidus projection surface and isothermal section at $1000^{\circ} \mathrm{C}$ of the Co-Pr-B (Co-rich) ternary phase diagram. J. Phas. Equilibria 14, 31 (1993).

[25] E.N. Kablov, V.P. Piskorskii, R.A. Valeev, O.G. Ospennikova, I.I. Rezchikova, N.S. Moiseeva. Role of interphase boron diffusion in the formation of the magnetic properties of sintered (Pr, Dy)-(Fe, Co)-B materials. J. Rus. Metallurgy (Metally) 7, 547 (2014).

[26] M. Sagawa, S. Fujimura, H. Yamamoto, Y. Matsuura. Permanent magnet materials based on the rare earth-iron-boron tetragonal compounds. IEEE Trans. Magn. 20, 1584 (1984).

[27] Z.W. Liu, H.A. Davies. Influence of Co substitution for Fe on the magnetic properties of nanocrystalline (Nd,Pr)-Fe-B based alloys. J. Phys. D: Appl. Phys. 39, 2647 (2006).

[28] R. Krishnan, L. Driouch, F.E. Kayzel, J.J.J.M. Franse. Magnetic coupling in amorphous Co-Dy-B and Fe-Dy-B alloys. Appl. Phys. Lett. 68, 256 (1996).

[29] J.J. Liu, W.J. Ren, J. Li, X.G. Zhao, W. Liu, Z.D. Zhang. High Pr-content $\left(\mathrm{Tb}_{0.2} \mathrm{Pr}_{0.8}\right)\left(\mathrm{Fe}_{0.4} \mathrm{Co}_{0.6}\right)_{1.93-\mathrm{x}} \mathrm{B}_{\mathrm{x}}$ magnetostrictive alloys. Appl. Phys. Lett. 87, 082506 (2005). 